\title{
Mechanical Resonance Dispersion Revisited
}

\author{
Haskell V. Hart ${ }^{*}$ \\ 970 Janet Dr., Canyon Lake, TX 78133, USA
}

\begin{abstract}
Mechanical resonance dispersion is the inelastic response of a solid to a periodic shear stress. Instead of the elastic Young's Modulus, the phenomenon is described by both a real J', and an imaginary J" component of complex shear compliance, corresponding to in phase and out of phase strain responses, respectively. The experimental results are plots of $\mathrm{J}^{\prime}$ and $\mathrm{J} " v s$. frequency, which are typically in the audiofrequency range of $10-5600 \mathrm{~Hz}$. Resonances are observed as maxima in $\mathrm{J}^{\prime}$ and inversions in $\mathrm{J}^{\prime}$ at frequencies corresponding to modes of plastic deformation, which are much lower frequencies (audiofrequency range) than elastic normal modes. The theoretical explanation of Edwin R. Fitzgerald involves particle waves and momentum transfer and leads to a particle-in-a-box frequency formula for these inelastic modes. Unfortunately, most of his and other published raw data were never analyzed by this model. The purpose of this article is to apply this formula to previously uninterpreted resonance dispersion curves and to address some of the earlier criticism of Fitzgerald's work. Results of these calculations support the Fitzgerald Theory to a high degree, demonstrate the importance of impurities and chemical analysis, largely mollify previous criticisms, and suggest the possibility of a new particle wave mass spectroscopy at great distances.
\end{abstract}

Keywords: Complex Shear Compliance, Mechanical Resonance Dispersion, Particle-in-a-box, Plasticity.

\section{INTRODUCTION}

Mechanical resonance dispersion (MRD) has been applied to such diverse systems as whale blubber [1], violin wood [2], human cancellous bone, natural rubber, synthetic polymers, and a variety of single crystal and polycrystalline solids, both elements and compounds (other references below in context), with the goal of understanding the mechanical response of the solid to periodic stress in the audiofrequency range. According to Edwin R. Fitzgerald [3], the complex (hence "dispersion") shear compliance, $J^{*}=J^{\prime}-i J^{\prime \prime}$, with $J^{\prime}$ equal to the (energy) storage compliance and $J^{\prime \prime}$ equal to the (energy) loss compliance. This is the manifestation of a periodic shear stress, $s=s_{0} \sin \omega t$, resulting in a periodic strain, $a$, with both in phase and out of phase components: $a=s_{0}\left(J^{\prime} \sin \omega t-J^{\prime \prime} \cos \omega t\right)$.

In a typical Fitzgerald experiment [3], the sample disk is clamped between piezoelectric transducers and is subjected to periodic shear stress at specific narrowly spaced frequencies. $\mathrm{J}^{\prime}$ and $\mathrm{J}^{\prime \prime}$ are calculated at each frequency from knowledge of the electrical circuit [1], yielding a "spectrum" for a particular material. The details of the spectrum are a function of the chemical composition, including impurities, the crystallinity, the previous processing (e.g., sintering, fusion, quenching, or annealing), and to a lesser extent the static stress on the sample and the duration of the experiment.

*Address correspondence to this author at the 970 Janet Dr., Canyon Lake, TX 78133, USA; Tel: 713-962-3848; E-mail: hvhart@swbell.net

\section{COMPUTATIONAL METHODS}

The classical mechanical model predicts a single resonance frequency, but nearly all spectra, including those of elements, show multiple resonances. To explain these and other details, Fitzgerald [4] invoked a momentum transfer equation for a one dimensional lattice of $\mathrm{N}$ atoms $(1,2, \ldots \mathrm{N})$ of mass $m$ and velocities, $v_{1}, v_{2}, \ldots, v_{n-1}, v_{n}, v_{n+1}, \ldots, v_{N}$.

$m \frac{\partial v_{n}}{\partial t}=K_{p}\left(v_{n+1}+v_{n-1}-2 v_{n}\right)$

where $K_{p}$ is the momentum transfer constant, found to be equal to $i h /\left(4 \pi d^{2}\right)$, with $h$ equal to Planck's Constant, $m$ the atomic mass, and $d$ the interatomic spacing.

Applying boundary conditions to standing particle wave solutions to (1), the Planck-Einstein Equation, $E=h v$, plus the deBroglie Relation, $p \lambda=h$, Fitzgerald derived the particle-in-a-box normal mode frequencies

$v=q^{2} h /\left(8 m S^{2}\right)$

from which we obtain the operational equation for interpreting MRD spectra,

$S=q(h / 8 m v)^{1 / 2}$

Here $S$ equals $N d$, the crystal mosaic size, and $q$ a quantum number $1,2, \ldots N-1$. A standing particle wave is thus fixed between the endpoints of the mosaic separated by $\mathrm{S}$ and can have $N-1$ wavelengths from $2 S$ to $2 d$. Since 
Table 1. Polycrystalline lead ${ }^{\mathrm{a}}$, Ref. [3].

\begin{tabular}{|c|c|c|c|c|}
\hline Isotope/Element & $v(\mathbf{H z})$ & $v_{\mathrm{C}}(\mathrm{Hz})$ & $q$ & $S(\mu \mathrm{m})$ \\
\hline \multicolumn{5}{|c|}{ Sample A } \\
\hline${ }^{44} \mathrm{Ca}$ & 975 & 977 & 1 & 1.08 \\
\hline $\mathrm{Ag}$ & 1600 & 1592 & 2 & 1.08 \\
\hline $\mathrm{Pb}$ & 1850 & 1865 & 3 & 1.08 \\
\hline OR Bi & & 1849 & 3 & 1.08 \\
\hline${ }^{65} \mathrm{Cu}$ & 2650 & 2645 & 2 & 1.08 \\
\hline${ }^{60} \mathrm{Ni}$ & 2875 & 2866 & 2 & 1.08 \\
\hline \multicolumn{5}{|c|}{ Sample A Annealed } \\
\hline $\mathrm{Cu}$ & 2900 & 2904 & 2 & 1.04 \\
\hline $\mathrm{Pb}$ & 3550 & 3363 & 4 & 1.04 \\
\hline OR Bi & & 3532 & 4 & 1.04 \\
\hline \multicolumn{5}{|c|}{ Sample $B^{b}$} \\
\hline $\mathrm{Cu}$ & 2825 & 2852 & 2 & 1.05 \\
\hline $\mathrm{Pb}$ & 3500 & 3498 & 4 & 1.05 \\
\hline OR Bi & & 3469 & 4 & 1.04 \\
\hline \multicolumn{5}{|c|}{ Sample B Annealed } \\
\hline $\mathrm{Pb}$ & 3550 & 3563 & 4 & 1.04 \\
\hline OR Bi & & 3532 & 4 & 1.04 \\
\hline \multicolumn{5}{|c|}{ Sample $C^{c}$} \\
\hline${ }^{65} \mathrm{Cu}$ & 2815 & 2887 & 2 & 1.04 \\
\hline $\mathrm{Pb}$ & 3650 & 3619 & 4 & 1.03 \\
\hline OR Bi & & 3588 & 4 & 1.02 \\
\hline
\end{tabular}

${ }^{\mathrm{a}}$ Chemical analysis: $\mathrm{Bi}<0.0001 \%$, Cu 0.0001\%, Fe 0.0002\%

${ }^{\mathrm{b}}$ Different dimensions

${ }^{c}$ Obtained from different location on bar, dimensions same as Sample A.

momentum is conserved in each of three dimensions, Eq. (1) is applicable to any row of like atoms in a crystal. An identical result, Eq. (2), was obtained [4, 5] by applying the timedependent Schrödinger Equation.

For many materials a common dimension for crystal mosaics is on the order of $1 \mu \mathrm{m}$. This adds a further criterion for correct spectral assignments according to Eq. (3): $S \simeq 1 \mu \mathrm{m}$. Of course, elements chosen, which determine $m$, must be known to be present in the sample or likely to be present based on sample origin and history.

Interpretation of a spectrum of resonance frequencies by this model will consist of choosing an element or an isotope for a highly resolved spectrum and a value of $q$ for each resonant frequency such that a common value of $S$ is calculated by Eq. (3) for all resonant frequencies in the spectrum.
This has been done only rarely previously for multiple resonances [6-9] where frequency series with ratios 1: 4: 9: $16 \ldots \mathrm{q}^{2}$ were found (twice retrospectively). Such a complete spectrum is not observed in any of the 38 spectra interpreted below, and never without additional resonances, hence the need to identify previously unrecognized sample impurities in the large majority of MRD spectra. The purpose of this article is to apply this formula (Eqs. (2) and (3)) to previously uninterpreted MRD spectra and to address some of the earlier criticism of Fitzgerald's work.

Experimental resonant frequencies $v$ listed in the following tables were either specifically mentioned in text or tables by the authors of the respective references or were taken from their spectra figures by this author. Eq. (3) was used to calculate $S$ from each frequency and the assigned values of atomic or isotopic mass and $q$. The average value of $S, S_{a v}$, for each sample was used to calculate $v_{c}$ from Eq. (2) for comparison with experimental values, $v$. Throughout the following tables, an element symbol implies no resolution of isotopes or a monoisotopic element and the use of the atomic weight [10] in determining $m$ for Eq. (3). A specific isotope designation implies use of the specific isotopic mass [11] in Eq. (3).

\section{RESULTS AND DISCUSSION}

\subsection{Metals}

\subsubsection{Elemental}

Table 1 lists data [3] from three different polycrystalline lead samples cut from the same bar. Samples A and C have the same dimensions, but were cut from different ends of the bar. Sample B was cut near A but had different dimensions.

The importance of trace impurities $(\mathrm{Cu}, \mathrm{Ag}, \mathrm{Ca}, \mathrm{Ni}$ and possibly $\mathrm{Bi}$ ) is made strikingly obvious by this interpretation. Resonances due to copper were the strongest. Silver, a common lead impurity, was not reported in the chemical analysis, but is indicated based on a weak resonance. Lead and bismuth resonances would probably not have been resolved, so both possibilities are listed. In the Betterton-Kroll refining process calcium is added to the molten lead to extract bismuth as dross $\left(\mathrm{Ca}_{3} \mathrm{Bi}_{2}\right)$. The origin of $\mathrm{Ni}$ is unknown. The good agreement between calculated $S$ values, all near one micron, supports the validity of Eq. (3) and the underlying theory. This degree of agreement or better will be seen throughout the following data sets for other materials. Notice that the annealed samples had fewer resonances, which may be the result of other impurities having moved to the grain boundaries. Spectral differences between the samples may be due to heterogeneity of the lead bar from which all samples were cut, orientation of the samples within the bar, or variation in time of analysis. Whether the free particles are atoms or ions is not known.

In a higher resolution study of lead [12], S. R. Bodner obtained the results in Table 2. Natural frequency (elastic) 
Table 2. Polycrystalline lead, Ref. [12].

\begin{tabular}{|c|c|c|c|c|}
\hline Isotope/Element & $v(\mathbf{H z})$ & $v_{\mathrm{C}}(\mathrm{Hz})$ & $q$ & $S(\mu \mathrm{m})$ \\
\hline $\mathrm{Pb}_{6}$ & 15.9 & 14.7 & 1 & 1.59 \\
\hline $\mathrm{Pb}_{2}$ & 45.5 & 44.0 & 1 & 1.63 \\
\hline $\mathrm{Pb}$ & 89.1 & 88.1 & 1 & 1.64 \\
\hline $\mathrm{Sb}$ & 146 & 150 & 1 & 1.68 \\
\hline $\mathrm{Br}$ & 221 & 228 & 1 & 1.68 \\
\hline $\mathrm{Fe}$ & 311 & 327 & 1 & 1.69 \\
\hline${ }^{44} \mathrm{Ca}$ & 412 & 415 & 1 & 1.66 \\
\hline $\mathrm{Cl}$ & 532 & 515 & 1 & 1.63 \\
\hline $\mathrm{Ag}$ & 661 & $678 \mathrm{E}^{\mathrm{a}}$ & 2 & 1.67 \\
\hline $\mathrm{Pb}$ & 808 & 795 & 3 & 1.64 \\
\hline As & 972 & 977 & 2 & 1.66 \\
\hline $\mathrm{Cu}$ & 1133 & 1134 & 2 & 1.65 \\
\hline $\mathrm{Sb}$ & 1313 & $1352 \mathrm{E}^{\mathrm{a}}$ & 3 & 1.68 \\
\hline $\mathrm{Ag}$ & 1512 & 1526 & 3 & 1.66 \\
\hline \multirow[t]{2}{*}{${ }^{44} \mathrm{Ca}$} & 1722 & 1665 & 2 & 1.62 \\
\hline & 1925 & $E^{b}$ & & \\
\hline As & 2170 & 2198 & 3 & 1.66 \\
\hline $\mathrm{Sb}$ & 2405 & 2404 & 4 & 1.65 \\
\hline $\mathrm{Cu}$ & 2685 & $2591 \mathrm{E}^{\mathrm{a}}$ & 3 & 1.62 \\
\hline OR Ag & 2685 & 2714 & 4 & 1.66 \\
\hline \multirow[t]{2}{*}{$\mathrm{Fe}$} & 2920 & 2948 & 3 & 1.66 \\
\hline & 3230 & $E^{b}$ & & \\
\hline
\end{tabular}

${ }^{\text {a }}$ Coincident Elastic natural frequency

${ }^{\mathrm{b}}$ Pure Elastic natural frequency

resonances as calculated by Bodner are intermixed, and are indicated by E. Here we see the whole range of lead impurities. All the listed metals and metalloids except calcium (mentioned above) occur naturally with lead. Lead chloride is added to remove the calcium. Bromide probably enters with the chloride. $\mathrm{Pb}_{2}$ is a known vapor phase molecule near the boiling point of lead (as are $\mathrm{Pb}_{3}$ and $\mathrm{Pb}_{4}$ ) [13]. $\mathrm{Pb}_{6}$ has not been reported, so this low frequency resonance may have another origin, especially since the corresponding value of $S$ is relatively low.

Also of importance is the fact that nonmetals do not have strong arc or spark (the techniques of the time) atomic emission spectra as do metals and metalloids, because of their tendency to combine in molecules, e.g. as CN. Thus some impurities listed in tables below would not have been seen in a typical elemental analysis. Examples are F, Cl, Br, I, C (the electrodes are graphite), N, O, P, S. Metalloids, e.g. As and
Table 3. Polycrystalline indium, ${ }^{\mathrm{a}}$ Ref. $[3]$.

\begin{tabular}{|c|c|c|c|c|}
\hline Element & $\boldsymbol{v}(\mathbf{H z})$ & $\boldsymbol{v}_{c}(\mathbf{H z})$ & $\boldsymbol{q}$ & $\boldsymbol{S}(\boldsymbol{\mu} \mathbf{m})$ \\
\hline \hline $\mathrm{In}$ & 1540 & 1566 & 2 & 1.06 \\
\hline OR Sn & & 1515 & 2 & 1.04 \\
\hline $\mathrm{Cu}$ & 2830 & 2830 & 2 & 1.05 \\
\hline
\end{tabular}

${ }^{a}$ Chemical analysis: $\mathrm{Cu} 0.006 \%, \mathrm{Zn} 0.01 \%$, Sn $0.01 \%$, Pb trace

Table 4. Polycrystalline aluminum, ${ }^{\mathrm{a}}$ Ref. [3].

\begin{tabular}{|c|c|c|c|c|}
\hline Element & $\boldsymbol{v}(\mathbf{H z})$ & $\boldsymbol{v}_{\mathbf{C}}(\mathbf{H z})$ & $\boldsymbol{q}$ & $\boldsymbol{S}(\boldsymbol{\mu} \mathbf{m})$ \\
\hline \hline $\mathrm{P}$ & 1315 & 1322 & 1 & 1.11 \\
\hline $\mathrm{Si}$ & 1435 & 1458 & 1 & 1.11 \\
\hline $\mathrm{Rb}$ & 1940 & 1916 & 2 & 1.10 \\
\hline $\mathrm{Fe}$ & 2890 & 2932 & 2 & 1.11 \\
\hline $\mathrm{Ti}$ & 3500 & 3421 & 2 & 1.09 \\
\hline
\end{tabular}

${ }^{\mathrm{a}}$ Chemical analysis: $\mathrm{Fe}, \mathrm{Si}, \mathrm{Cu}<0.002 \%$

$\mathrm{Sb}$, have typically weak emission lines compared to metals, so their detection limits would be higher.

Polycrystalline indium results are listed in Table 3 [3]. Again the copper impurity had the strongest resonance; in this case it was a known impurity. Due to its nearly equal atomic mass, the tin impurity resonance, if present, was not resolved from that of indium.

Data for polycrystalline aluminum [3] are presented in Table 4. In addition to the listed impurities silicon and iron, sodium and titanium are also indicated. Common minerals associated with bauxite, the chief source of aluminum, are goethite $(\mathrm{FeO}(\mathrm{OH}))$, hematite $\left(\mathrm{Fe}_{2} \mathrm{O}_{3}\right)$, kaolinite $\left(\mathrm{Al}_{2} \mathrm{Si}_{2} \mathrm{O}_{5}(\mathrm{OH})_{2}\right)$, and anatase $\left(\mathrm{TiO}_{2}\right)$. Sodium enters the aluminum process as sodium hydroxide or cryolite, $\mathrm{Na}_{3} \mathrm{AlF}_{6}$, a flux. Phosphorous is found in kaolinite. Thus we see the likely sources of each impurity element except $\mathrm{Rb}$.

\subsubsection{Alloys}

Tables 5, 6, and $\mathbf{7}$ show spectral data for aluminum alloys $2 \mathrm{~S}$ (1100) and 3S (3003) from Bodner. Aluminum is hardened by addition of $\mathrm{Mg}, \mathrm{Ti}, \mathrm{V}, \mathrm{Mn}, \mathrm{Fe}, \mathrm{Cu}$, and $\mathrm{Zn}$, which in various combinations form separate precipitate phases. Spectra of 2 S (1100) and 3S (3003) aluminum alloys show three phases with different $S$-values: a primary (Al) phase with $S$ $\approx 1.47 \mu \mathrm{m}$ av., a Mn-rich precipitate phase with $S \approx 1.30 \mu \mathrm{m}$ av., and another containing the other elements with $S \approx 1.65$ $\mu \mathrm{m}$ av. This is consistent with microscopy studies. The silver entered with copper or lead, but the source of the lead is unknown. $\mathrm{Ag}_{3}$ is a known molecule in the vapor phase near the silver boiling point [15] and as a cluster in the crystalline phase, where it is stabilized by substituted acetylene ligands. [16] Field-free regions may well resemble gas phases as far as the stability of atoms, ions, and molecules goes. 
Table 5. 2S (1100) aluminum alloy data from Ref. [12].

\begin{tabular}{|c|c|c|c|c|}
\hline Isotope/Element & $\boldsymbol{v}(\mathbf{H z})$ & $\boldsymbol{v}_{\mathbf{C}}(\mathbf{H z})$ & $\boldsymbol{q}$ & $\boldsymbol{S}(\boldsymbol{\mu} \mathbf{m})$ \\
\hline \hline $\mathrm{Ag}_{3}$ & 57 & 57 & 1 & 1.64 \\
\hline $\mathrm{Ag}$ & 164 & 171 & 1 & 1.68 \\
\hline $\mathrm{Fe}$ & 333 & 330 & 1 & 1.64 \\
\hline $\mathrm{Mn}$ & 562 & 553 & 1 & 1.27 \\
\hline $\mathrm{Al}$ & 852 & 854 & 1 & 1.47 \\
\hline $\mathrm{Cu}$ & 1192 & 1162 & 2 & 1.62 \\
\hline $\mathrm{Ti}$ & 1588 & 1542 & 2 & 1.62 \\
\hline $\mathrm{Ni}$ & 2038 & 2059 & 2 & 1.29 \\
\hline $\mathrm{Cu}$ & 2545 & 2614 & 3 & 1.67 \\
\hline${ }^{24} \mathrm{Mg}$ & 3100 & 3077 & 2 & 1.64 \\
\hline${ }^{25} \mathrm{Mg}$ & 3695 & 3688 & 2 & 1.47 \\
\hline
\end{tabular}

${ }^{a} 1100$ is the new designation for $2 \mathrm{~S}$ aluminum.

Table 6. 2S (1100) aluminum from Ref. [14].

\begin{tabular}{|c|c|c|c|c|}
\hline Isotope/Element & $\boldsymbol{v}(\mathbf{H z})$ & $\boldsymbol{v}_{\mathrm{c}}(\mathbf{H z})$ & $\boldsymbol{q}$ & $\boldsymbol{S}(\boldsymbol{\mu \mathbf { m }})$ \\
\hline \hline $\mathrm{Pb}$ & 111 & 112 & 1 & 1.47 \\
\hline $\mathrm{Cu}$ & 295 & 294 & 1 & 1.63 \\
\hline $\mathrm{Mn}$ & 545 & 550 & 1 & 1.29 \\
\hline $\mathrm{Al}$ & 860 & 858 & 1 & 1.47 \\
\hline $\mathrm{Cu}$ & 1160 & 1177 & 2 & 1.65 \\
\hline $\mathrm{V}$ & 1430 & 1468 & 2 & 1.65 \\
\hline $\mathrm{Ti}$ & 1950 & 1934 & 2 & 1.46 \\
\hline $\mathrm{Mn}$ & 2220 & 2200 & 2 & 1.28 \\
\hline $\mathrm{Cu}$ & 2750 & 2647 & 3 & 1.60 \\
\hline${ }^{26} \mathrm{Mg}$ & 3550 & $3564 \mathrm{E}^{\mathrm{a}}$ & 2 & 1.47 \\
\hline
\end{tabular}

${ }^{\mathrm{a}}$ Coincident Elastic resonance.

Data [17] for coinage metals in US currency are listed in Table 8. These are polycrystalline alloys. The experimental apparatus here was slightly different, having a pick up (phonograph type) needle to detect resonances. The spectra were extremely simple, and there were no resonances for tin or nickel, both known to be present in significant amounts. Lead, a common silver impurity, appears in the dime. With only one resonance each, the penny and nickel spectra (2900 $\mathrm{Hz}$, and $2800 \mathrm{~Hz}$, respectively) could be fit with any element. For consistency with the dime resonance at $2700 \mathrm{~Hz}$, copper was selected.

\subsection{Single Crystal Salts}

Single crystal dispersion data for salts are listed in Tables 9-11. No chemical analyses were given in the original refer-
Table 7. 3S (3003) aluminum data from Ref.[12].

\begin{tabular}{|c|c|c|c|c|}
\hline Element & $\boldsymbol{v}(\mathbf{H z})$ & $\boldsymbol{v}_{\mathrm{C}}(\mathbf{H z})$ & $\boldsymbol{q}$ & $\boldsymbol{S}(\boldsymbol{\mu} \mathbf{m})$ \\
\hline \hline $\mathrm{Ag}$ & 170 & 164 & 1 & 1.65 \\
\hline $\mathrm{Mn}$ & 503 & 497 & 1 & 1.34 \\
\hline $\mathrm{Cu}$ & 1095 & 1117 & 2 & 1.69 \\
\hline $\mathrm{Zn}$ & 1650 & 1670 & 2 & 1.36 \\
\hline $\mathrm{Cu}$ & 2480 & 2513 & 3 & 1.69 \\
\hline
\end{tabular}

a 3003 is the new designation for $3 \mathrm{~S}$ aluminum.

Table 8. Coinage metals, Ref. [17].

\begin{tabular}{|c|c|c|c|c|}
\hline Element & $v(\mathbf{H z})$ & $v_{\mathrm{C}}(\mathrm{Hz})$ & $q$ & $S(\mu \mathrm{m})$ \\
\hline \multicolumn{5}{|c|}{ Penny $^{\mathrm{a}}$} \\
\hline $\mathrm{Cu}$ & 2900 & 2900 & 2 & 1.04 \\
\hline \multicolumn{5}{|c|}{ Nickel $^{\mathrm{b}}$} \\
\hline $\mathrm{Cu}$ & 2800 & 2800 & 2 & 1.06 \\
\hline \multicolumn{5}{|c|}{ Dime $^{\mathrm{c}}$} \\
\hline $\mathrm{Pb}$ & 1900 & 1881 & 3 & 1.07 \\
\hline $\mathrm{Cu}$ & 2700 & 2727 & 2 & 1.08 \\
\hline
\end{tabular}

Table 9. Copper sulfate single crystal, Ref. [18].

\begin{tabular}{|c|c|c|c|c|}
\hline Element/Ion & $\boldsymbol{v}(\mathbf{H z})$ & $\boldsymbol{v}_{\mathrm{C}}(\mathbf{H z})$ & $\boldsymbol{q}$ & $\boldsymbol{S}(\boldsymbol{\mu m})$ \\
\hline \hline $\mathrm{Cu}$ & 730 & 749 & 1 & 1.04 \\
\hline $\mathrm{S}^{\mathrm{a}}$ & 1515 & 1483 & 1 & 1.01 \\
\hline $\mathrm{Cu}$ & 2965 & 2994 & 2 & 1.03 \\
\hline $\mathrm{SO}_{4}^{2-}$ & 4520 & 4456 & 3 & 1.02 \\
\hline
\end{tabular}

${ }^{\text {a }}$ Could be $\mathrm{S}$ atom or $\mathrm{S}^{2-}$ ion.

ence [18]. Data for copper sulfate sheared along [001] appears in Table 9. In addition to copper, atomic sulfur and sulfate ion were found. The atomic sulfur resonance has an analogous precedent: atomic phosphorus in human cancellous bone [6,7]. However, the appearance of sulfate ion $\left(\mathrm{SO}_{4}{ }^{2-}\right)$ is of interest here, as a phosphate $\left(\mathrm{PO}_{4}{ }^{3-}\right)$ resonance was not observed in bone. The original Fitzgerald theory dealt with a monatomic solid, such as a metal. The assignment of ions was not addressed.

Rochelle Salt, sodium potassium tartrate [18], shows isotopic resolution and numerous impurity resonances (Table 10) in the high temperature phase at $25.5^{\circ} \mathrm{C}$. Shearing was 
Table 10. Rochelle Salt single crystal, Ref. [18].

\begin{tabular}{|c|c|c|c|c|}
\hline Isotope/Element & $v(\mathrm{~Hz})$ & $v_{\mathrm{C}}(\mathrm{Hz})$ & $q$ & $S(\mu \mathrm{m})$ \\
\hline \multicolumn{5}{|c|}{$25.5^{\circ} \mathrm{C}$ phase } \\
\hline $\mathrm{Ag}$ & 440 & 420 & 1 & 1.03 \\
\hline${ }^{65} \mathrm{Cu}$ & 665 & 698 & 1 & 1.07 \\
\hline $\mathrm{Ni}$ & 760 & 773 & 1 & 1.06 \\
\hline${ }^{57} \mathrm{Fe}$ & 800 & 796 & 1 & 1.05 \\
\hline${ }^{54} \mathrm{Fe}$ & 850 & 841 & 1 & 1.04 \\
\hline $\mathrm{Cr}$ & 875 & 872 & 1 & 1.05 \\
\hline${ }^{29,}{ }^{30} \mathrm{Si}$ & 1525 & 1539 & 1 & 1.05 \\
\hline $\mathrm{Ag}$ & 1720 & 1681 & 2 & 1.04 \\
\hline $\mathrm{Na}$ & 1980 & 1972 & 1 & 1.05 \\
\hline${ }^{65} \mathrm{Cu}$ & 2720 & 2794 & 2 & 1.06 \\
\hline${ }^{63} \mathrm{Cu}$ & 2910 & 2882 & 2 & 1.04 \\
\hline $\mathrm{Fe}$ & 3250 & 3248 & 2 & 1.05 \\
\hline $\mathrm{V}$ & 3565 & 3561 & 2 & 1.05 \\
\hline \multicolumn{5}{|c|}{$20.2^{\circ} \mathrm{C}$ phase } \\
\hline $\mathrm{Ag}$ & 440 & 420 & 1 & 1.03 \\
\hline${ }^{41} \mathrm{~K}$ & 1125 & 1107 & 1 & 1.04 \\
\hline${ }^{39} \mathrm{~K}$ & 1210 & 1164 & 1 & 1.03 \\
\hline${ }^{29,30} \mathrm{Si}$ & 1535 & 1539 & 1 & 1.05 \\
\hline $\mathrm{Ag}$ & 1735 & 1681 & 2 & 1.03 \\
\hline $\mathrm{Na}$ & 2000 & 1972 & 1 & 1.04 \\
\hline${ }^{65} \mathrm{Cu}$ & 2815 & 2794 & 2 & 1.04 \\
\hline${ }^{63} \mathrm{Cu}$ & 2910 & 2882 & 2 & 1.04 \\
\hline $\mathrm{Fe}$ & 3300 & 3248 & 2 & 1.04 \\
\hline $\mathrm{V}$ & 3620 & 3561 & 2 & 1.04 \\
\hline
\end{tabular}

along [010] of the orthorhombic form. Due to the use of steel and copper vessels, iron and copper are known impurities in cream of tartar (potassium bitartrate), the industrial precursor of Rochelle Salt [19]. Silver is a common copper impurity. Silicon, vanadium, chromium, and nickel are likely elements in the stainless steel. Potassium sulfate is used in the purification process. Hence we understand the origin of all the impurities in Table $\mathbf{1 0}$.

A lower temperature phase $\left(20.2^{\circ} \mathrm{C}\right)$ was also studied. Interestingly, when the temperature was returned from $20.2^{\circ} \mathrm{C}$ to $26^{\circ} \mathrm{C}$, the spectrum did not match the original at $25.5^{\circ} \mathrm{C}$, but remained like that at $20.2^{\circ} \mathrm{C}$. We see this, and other similar spectrum simplifications with time, as evidence to support the presence of impurities which move to dislocations or grain boundaries when the sample is stressed over time. As
Table 11. Sodium chloride single crystal, Ref. [18].

\begin{tabular}{|c|c|c|c|c|}
\hline Isotope/Element & $\boldsymbol{v}(\mathbf{H z})$ & $\boldsymbol{v}_{\mathbf{C}}(\mathbf{H z})$ & $\boldsymbol{q}$ & $\boldsymbol{S}(\boldsymbol{\mu \mathbf { m }})$ \\
\hline \hline${ }^{81} \mathrm{Br}$ & 635 & 640 & 1 & 0.985 \\
\hline${ }^{34} \mathrm{~S}$ & 1520 & 1525 & 1 & 0.983 \\
\hline${ }^{32} \mathrm{~S}$ & 1640 & 1620 & 1 & 0.975 \\
\hline $\mathrm{Al}$ & 1950 & 1920 & 1 & 0.974 \\
\hline${ }^{79} \mathrm{Br}$ & 2650 & 2625 & 2 & 0.977 \\
\hline $\mathrm{F}$ & 2680 & 2726 & 1 & 0.990 \\
\hline $\mathrm{Ge}$ & 2875 & 2852 & 2 & 0.977 \\
\hline${ }^{18} \mathrm{O}$ & 2890 & 2878 & 1 & 0.979 \\
\hline $\mathrm{OR} \mathrm{H}_{2} \mathrm{O}$ & & 2875 & 1 & 0.979 \\
\hline${ }^{16} \mathrm{O}$ & 3210 & 3238 & 1 & 0.986 \\
\hline${ }_{\mathrm{Ni}}^{\mathrm{Fe}}$ & 3470 & 3530 & 2 & 0.990 \\
\hline $\mathrm{Sc}$ & 3700 & 3710 & 2 & 0.983 \\
\hline${ }^{41} \mathrm{~K}$ & 5000 & 5058 & 2 & 0.987 \\
\hline${ }^{39} \mathrm{~K}$ & 5370 & 5317 & 2 & 0.976 \\
\hline
\end{tabular}

Table 12. Sodium chloride single crystal, Ref. [20].

\begin{tabular}{|c|c|c|c|c|}
\hline Element/Isotope & $\boldsymbol{v}(\mathbf{H z})$ & $\boldsymbol{v}_{\mathbf{C}}(\mathbf{H z})$ & $\boldsymbol{q}$ & $\boldsymbol{S}(\boldsymbol{\mu m})$ \\
\hline \hline $\mathrm{Br}$ & 600 & 599 & 1 & 1.02 \\
\hline${ }^{37} \mathrm{Cl}$ & 1300 & 1295 & 1 & 1.02 \\
\hline $\mathrm{I}$ & 1500 & 1509 & 2 & 1.02 \\
\hline $\mathrm{Mg}$ & 2000 & 1970 & 1 & 1.01 \\
\hline $\mathrm{Na}$ & 2100 & 2083 & 1 & 1.02 \\
\hline $\mathrm{Br}$ & 2400 & 2397 & 2 & 1.02 \\
\hline${ }^{63} \mathrm{Cu}$ & 3100 & 3043 & 2 & 1.01 \\
\hline $\mathrm{I}$ & 3300 & 3396 & 3 & 1.04 \\
\hline${ }^{44} \mathrm{Ca}$ & 4300 & 4357 & 2 & 1.03 \\
\hline
\end{tabular}

some impurities are removed, others are stimulated in a sequence determined more by crystal energetics than phase transitions. Specific isotopes were resolved in this and some subsequent materials.

Table 11 presents data [18] for single crystal sodium chloride with shear applied in the [100] direction of the fcc crystal. Data from three different times and spectral regions in the original reference were combined. Germanium and scandium are unusual. The former may be from an electrical component of the apparatus. Water is a possible impurity.

Table 12 lists sodium chloride data from another source. [20] The importance of source and chemical analysis is again demonstrated. 
Table 13. Sodium chloride single crystal, Ref. [21].

\begin{tabular}{|c|c|c|c|c|}
\hline Isotope/Element & $\boldsymbol{v}(\mathrm{Hz})$ & $\boldsymbol{v}_{\mathrm{C}}(\mathbf{H z})$ & $\boldsymbol{q}$ & $\boldsymbol{S}(\boldsymbol{\mu m})$ \\
\hline \hline \multicolumn{5}{|c|}{ Sample A: Freshly cleaved } \\
\hline${ }^{41} \mathrm{~K}$ & 880 & 896 & 1 & 1.18 \\
\hline${ }^{39} \mathrm{~K}$ & 950 & 942 & 1 & 1.16 \\
\hline $\mathrm{Na}$ & 1600 & 1596 & 1 & 1.16 \\
\hline${ }^{37} \mathrm{Cl}$ & 4000 & 3971 & 2 & 1.16 \\
\hline \multicolumn{5}{|c|}{ Sample B: As received, Electrode 1 } \\
\hline${ }^{39} \mathrm{~K}$ & 1100 & 1093 & 1 & 1.08 \\
\hline${ }^{\mathrm{Na}}$ & 1900 & 1853 & 1 & 1.07 \\
\hline${ }^{81} \mathrm{Br}$ & 2050 & 2106 & 2 & 1.10 \\
\hline${ }^{79} \mathrm{Br}$ & 2150 & 2159 & 2 & 1.08 \\
\hline \multicolumn{7}{|c|}{ Sample B: Freshly cleaved, Electrode 2 } \\
\hline${ }^{44} \mathrm{Ca}$ & 850 & 848 & 1 & 1.16 \\
\hline${ }^{39} \mathrm{~K}$ & 950 & 957 & 1 & 1.16 \\
\hline $\mathrm{Na}$ & 1600 & 1622 & 1 & 1.16 \\
\hline & 1925 & 1890 & 2 & 1.15 \\
\hline
\end{tabular}

Table 14. Potassium bromide single crystal, Ref. [20], as cleaved $31.3 \mathrm{~g} / \mathrm{mm}^{2}$ static stress.

\begin{tabular}{|c|c|c|c|c|}
\hline Isotope/Element & $\boldsymbol{v}(\mathbf{H z})$ & $\boldsymbol{v}_{\mathrm{C}}(\mathbf{H z})$ & $\boldsymbol{q}$ & $\boldsymbol{S}(\boldsymbol{\mu m})$ \\
\hline \hline $\mathrm{Na}$ & 1250 & 1292 & 1 & 1.32 \\
\hline $\mathrm{Br}$ & 1500 & 1486 & 2 & 1.29 \\
\hline $\mathrm{K}$ & 2950 & 3038 & 2 & 1.32 \\
\hline${ }^{37} \mathrm{Cl}$ & 3275 & 3213 & 2 & 1.28 \\
\hline${ }^{35} \mathrm{Cl}$ & 3440 & 3397 & 2 & 1.29 \\
\hline $\mathrm{Al}$ & 4500 & 4402 & 2 & 1.28 \\
\hline
\end{tabular}

Single crystal sodium chloride data from yet another source [21] are listed in Table 13. The apparatus here is similar to that employed above for coinage metals (Table 4) in that it employed two pick up needles (electrodes). Results of two electrodes are combined in Sample A. In Sample B Electrode 1 was on an original surface, while electrode 2 was on a freshly cleaved surface. The freshly cleaved surfaces had a higher value of $S_{a v}$. The ultimate source of sodium chloride is the ocean, which is relatively rich in $\mathrm{Mg}, \mathrm{Ca}, \mathrm{S}$, and $\mathrm{K}$, with much less $\mathrm{Fe}, \mathrm{Cu}, \mathrm{F}, \mathrm{Br}$, and I [22], all of which are present in Tables 11, 12, and $\mathbf{1 3}$.

Tables 14 and $\mathbf{1 5}$ show data for potassium bromide under two different static stresses [20]. Impurities vary, which may be due to impurity particles entering the field free region in a
Table 15. Potassium bromide single crystal, Ref. [20], as cleaved, $8.9 \mathrm{~g} / \mathrm{mm}^{2}$ static stress.

\begin{tabular}{|c|c|c|c|c|}
\hline Isotope & $\boldsymbol{v}(\mathbf{H z})$ & $\boldsymbol{v}_{\mathrm{C}}(\mathbf{H z})$ & $\boldsymbol{q}$ & $\boldsymbol{S}(\boldsymbol{\mu m})$ \\
\hline \hline${ }^{29} \mathrm{Si}$ & 1000 & 1000 & 1 & 1.31 \\
\hline${ }^{41} \mathrm{~K}$ & 2800 & 2829 & 2 & 1.32 \\
\hline${ }^{39} \mathrm{~K}$ & 3000 & 2974 & 2 & 1.31 \\
\hline${ }^{37} \mathrm{Cl}$ & 3150 & 3134 & 2 & 1.31 \\
\hline${ }^{35} \mathrm{Cl}$ & 3300 & 3313 & 2 & 1.31 \\
\hline
\end{tabular}

Table 16. Potassium chloride single crystal, Ref. [20], annealed, $40.5 \mathrm{~g} / \mathrm{mm}^{2}$.

\begin{tabular}{|c|c|c|c|c|}
\hline Element/Isotope & $\boldsymbol{v}(\mathbf{H z})$ & $\boldsymbol{v}_{\mathrm{C}}(\mathbf{H z})$ & $\boldsymbol{q}$ & $\boldsymbol{S}(\boldsymbol{\mu m})$ \\
\hline \hline $\mathrm{Br}$ & 1500 & 1483 & 2 & 1.29 \\
\hline${ }^{41} \mathrm{~K}$ & 2850 & 2893 & 2 & 1.31 \\
\hline${ }^{39} \mathrm{~K}$ & 3050 & 3042 & 2 & 1.30 \\
\hline${ }^{37} \mathrm{Cl}$ & 3200 & 3206 & 2 & 1.30 \\
\hline${ }^{35} \mathrm{Cl}$ & 3400 & 3389 & 2 & 1.30 \\
\hline
\end{tabular}

Table 17. Lithium fluoride single crystal, Ref. [20], annealed, $28.9 \mathrm{~g} / \mathrm{mm}^{2}$.

\begin{tabular}{|c|c|c|c|c|}
\hline Isotope/Element & $\boldsymbol{v}(\mathbf{H z})$ & $\boldsymbol{v}_{\mathrm{C}}(\mathbf{H z})$ & $\boldsymbol{q}$ & $\boldsymbol{S}(\boldsymbol{\mu \mathbf { m }})$ \\
\hline \hline${ }^{18} \mathrm{O}$ & 1200 & 1177 & 1 & 1.52 \\
\hline $\mathrm{OR} \mathrm{H}_{2} \mathrm{O}$ & & 1176 & 1 & 1.52 \\
\hline $\mathrm{Fe}$ & 1500 & 1518 & 2 & 1.54 \\
\hline${ }^{7} \mathrm{Li}$ & 2950 & 3020 & 1 & 1.55 \\
\hline${ }^{6} \mathrm{Li}$ & 3450 & 3522 & 1 & 1.55 \\
\hline $\mathrm{Na}$ & 3750 & 3686 & 2 & 1.52 \\
\hline
\end{tabular}

sequence determined in part by external stress, especially as it affects dislocation arrays [20]. Rather than refer to resonance peaks as "shifting" to higher frequencies with increased static stress, we see here that some frequencies disappear and others emerge, as the natural result of new impurity particles being dislodged to the field free region. There remains, however, the lesser effect of decreasing mosaic size with increased stress, which shifts persisting resonances to higher frequency.

Tables 16 and $\mathbf{1 7}$ show data for potassium chloride and lithium fluoride, respectively [20]. These alkali halide salts show the familiar pattern of alkali metal and halide impurities (equal charge substituents) seen above, plus iron in lithium fluoride. The impurities seen in all [20] alkali halide salts are typical for these materials. 
Table 18. Quartz single crystal $^{\text {a }}$ sheared $\perp$ to c-axis, Ref. [23].

\begin{tabular}{|c|c|c|c|c|}
\hline Isotope/Element & $\boldsymbol{v}(\mathbf{H z})$ & $\boldsymbol{v}_{\mathrm{C}}(\mathbf{H z})$ & $\boldsymbol{q}$ & $\boldsymbol{S}(\boldsymbol{\mu m})$ \\
\hline \hline $\mathrm{P}$ & 1250 & 1251 & 1 & 1.14 \\
\hline${ }^{30} \mathrm{Si}$ & 1300 & 1293 & 1 & 1.13 \\
\hline $\mathrm{Fe}$ & 2760 & 2776 & 2 & 1.14 \\
\hline $\mathrm{V}$ & 3025 & 3043 & 2 & 1.14 \\
\hline $\mathrm{Ti}$ & 3260 & 3238 & 2 & 1.13 \\
\hline
\end{tabular}

${ }^{\mathrm{a}}$ Source: Minas Geraes, Brazil.

Table 19. Quartz single crystal $^{\mathrm{a}}$ sheared $\|$ to c-axis, Ref. [23].

\begin{tabular}{|c|c|c|c|c|}
\hline Isotope/Element & $\boldsymbol{v}(\mathbf{H z})$ & $\boldsymbol{v}_{\mathbf{C}}(\mathbf{H z})$ & $\boldsymbol{q}$ & $\boldsymbol{S}(\boldsymbol{\mu m})$ \\
\hline \hline $\mathrm{P}$ & 1085 & 1091 & 1 & 1.22 \\
\hline $\mathrm{Na}$ & 1455 & 1470 & 1 & 1.22 \\
\hline $\mathrm{W}$ & 1625 & 1654 & 3 & 1.23 \\
\hline $\mathrm{Bi}$ & 2565 & 2587 & 4 & 1.22 \\
\hline $\mathrm{Ti}$ & 2865 & 2824 & 2 & 1.21 \\
\hline${ }^{44} \mathrm{Ca}$ & 3150 & 3075 & 2 & 1.20 \\
\hline $\mathrm{Be}$ & 3840 & 3750 & 1 & 1.20 \\
\hline${ }^{35} \mathrm{Cl}$ & 3890 & 3866 & 2 & 1.21 \\
\hline $\mathrm{P}$ & 4480 & 4364 & 2 & 1.20 \\
\hline${ }^{30} \mathrm{Si}$ & 4540 & 4510 & 2 & 1.21 \\
\hline${ }^{29} \mathrm{Si}$ & 4600 & 4665 & 2 & 1.22 \\
\hline${ }^{65} \mathrm{Cu}$ & 4630 & 4685 & 3 & 1.22 \\
\hline${ }^{28} \mathrm{Si}$ & 4700 & 4832 & 2 & 1.23 \\
\hline${ }^{63} \mathrm{Cu}$ & 4780 & 4833 & 3 & 1.22 \\
\hline $\mathrm{Mg}$ & 5600 & 5562 & 2 & 1.21 \\
\hline
\end{tabular}

${ }^{\text {a }}$ Same source as Table $\mathbf{1 8}$.

\subsection{Natural Polymers}

\subsubsection{Quartz}

Quartz $\left(\mathrm{SiO}_{2}\right)$ is a naturally occurring three-dimensional polymer. Tables 18 and 19 show dispersion data [23] for single crystals from the same source sheared $\perp$ and $\|$ to the hexagonal c-axis, respectively. Chemical analysis showed Fe 0.01\%, Al 0.004\%, Mg 0.0001\%, Ca 0.002\%, Li and $\mathrm{Bi}$ possible trace. All of the above elements, except $\mathrm{Al}$ were found in the resonances, plus $\mathrm{Si}, \mathrm{P}, \mathrm{Na}$, and $\mathrm{K}$. We expect $\mathrm{Si}$. Ti and $\mathrm{W}$ are also tetravalent. $\mathrm{P}$ balances $\mathrm{Al}$ as substituents for $\mathrm{Si}$, as they have one more and one less valence electron, respectively. In fact, $\mathrm{AlPO}_{4}$ is isostructural with quartz with almost the same bond distances, $\mathrm{Si}-\mathrm{O}$, vs. Al-O and P-O. Trivalent $\mathrm{Fe}(\mathrm{III})$ is an alternative to $\mathrm{Al}$, while one $\mathrm{Ca}$, one $\mathrm{Mg}$, or one $\mathrm{Fe}(\mathrm{II})$ atom (all divalent) requires two P's for balance of charge in the quartz matrix.

Table 20 lists resonances for fused quartz samples of three different thicknesses, all cut from the same rod [23]. Chemical analysis showed $\mathrm{Fe} 0.02 \%, \mathrm{Al} 0.004 \%, \mathrm{Mg}$ $0.0001 \%$, Ca $0.002 \%, \mathrm{Cu} 0.0005 \%$, Cr $0.004 \%$, Mn 0.001\%, Li $0.001 \%$, K possible trace. All the listed impurities showed up except Li, Al and Mn, plus P, S, Cl, Sc, Ti, V, Ni, Rb, W and the ubiquitous $\mathrm{Na} . \mathrm{P}, \mathrm{Ti}, \mathrm{V}$, and $\mathrm{W}$ are quadrivalent and could substitute for Si. They are also common elements in steel, as are $\mathrm{Cu}, \mathrm{Cr}$ and $\mathrm{Ni}$, suggesting that these elements may have been introduced in the fusion process.

Table 20. Fused quartz, ${ }^{\mathrm{a}}$ Ref. [23].

\begin{tabular}{|c|c|c|c|c|}
\hline Isotope/Element & $v(\mathbf{H z})$ & $v_{\mathrm{C}}(\mathrm{Hz})$ & $q$ & $S(\mu \mathrm{m})$ \\
\hline \multicolumn{5}{|c|}{ Sample A, 0.199 in $^{b}$} \\
\hline $\mathrm{P}$ & 1315 & 1311 & 1 & 1.11 \\
\hline${ }^{25} \mathrm{Mg}$ & 1600 & 1625 & 1 & 1.12 \\
\hline${ }^{24} \mathrm{Mg}$ & 1695 & 1692 & 1 & 1.11 \\
\hline W & 2040 & 1987 & 3 & 1.09 \\
\hline${ }^{65} \mathrm{Cu}$ & 2495 & 2501 & 2 & 1.11 \\
\hline $\mathrm{Ni}$ & 2730 & 2766 & 2 & 1.12 \\
\hline${ }^{57} \mathrm{Fe}$ & 2820 & 2852 & 2 & 1.11 \\
\hline $\mathrm{Cr}$ & 3120 & 3123 & 2 & 1.11 \\
\hline $\mathrm{Ti}$ & 3370 & 3392 & 2 & 1.11 \\
\hline${ }^{37} \mathrm{Cl}$ & 4480 & 4392 & 2 & 1.10 \\
\hline${ }^{32} \mathrm{~S}$ & 5135 & 5078 & 2 & 1.10 \\
\hline $\mathrm{P}$ & 5185 & 5242 & 2 & 1.11 \\
\hline${ }^{30} \mathrm{Si}$ & 5410 & 5417 & 2 & 1.11 \\
\hline \multicolumn{5}{|c|}{ Sample B, 0.151 in ${ }^{\mathrm{b}}$} \\
\hline $\mathrm{Ca}$ & 1050 & 1077 & 1 & 1.09 \\
\hline S & 1320 & 1346 & 1 & 1.09 \\
\hline $\mathrm{Si}$ & 1565 & 1536 & 1 & 1.07 \\
\hline $\mathrm{Rb}$ & 2030 & 2019 & 2 & 1.07 \\
\hline${ }^{63} \mathrm{Cu}$ & 2810 & 2743 & 2 & 1.06 \\
\hline $\mathrm{Ni}$ & 2940 & 2941 & 2 & 1.08 \\
\hline${ }^{54} \mathrm{Fe}$ & 3195 & 3200 & 2 & 1.08 \\
\hline${ }^{53} \mathrm{Cr}$ & 3270 & 3260 & 2 & 1.07 \\
\hline V & 3400 & 3388 & 2 & 1.07 \\
\hline
\end{tabular}


Table 20. contd...

\begin{tabular}{|c|c|c|c|c|}
\hline Isotope/Element & $v(\mathbf{H z})$ & $v_{\mathrm{C}}(\mathrm{Hz})$ & $q$ & $S(\mu \mathrm{m})$ \\
\hline $\mathrm{Ti}$ & 3580 & 3606 & 2 & 1.08 \\
\hline $\mathrm{Sc}$ & 3850 & 3839 & 2 & 1.07 \\
\hline${ }^{41} \mathrm{~K}$ & 4195 & 4214 & 2 & 1.08 \\
\hline $\mathrm{Ca}$ & 4300 & 4306 & 2 & 1.08 \\
\hline${ }^{39} \mathrm{~K}$ & 4440 & 4430 & 2 & 1.07 \\
\hline \multicolumn{5}{|c|}{ Sample C, 0.126 in $^{\mathrm{b}}$} \\
\hline${ }^{41} \mathrm{~K}$ & 1090 & 1097 & 1 & 1.06 \\
\hline${ }^{34} \mathrm{~S}$ & 1310 & 1322 & 1 & 1.06 \\
\hline${ }^{29} \mathrm{Si}$ & 1560 & 1551 & 1 & 1.05 \\
\hline${ }^{28} \mathrm{Si}$ & 1600 & 1606 & 1 & 1.06 \\
\hline${ }^{26} \mathrm{Mg}$ & 1760 & 1730 & 1 & 1.04 \\
\hline W & 2160 & 2200 & 3 & 1.06 \\
\hline $\mathrm{Ni}$ & 3030 & 3063 & 2 & 1.06 \\
\hline $\mathrm{Cr}$ & 3450 & 3458 & 2 & 1.05 \\
\hline V & 3590 & 3529 & 2 & 1.04 \\
\hline $\mathrm{Ti}$ & 3780 & 3756 & 2 & 1.05 \\
\hline${ }^{44} \mathrm{Ca}$ & 4100 & 4090 & 2 & 1.05 \\
\hline${ }^{40} \mathrm{Ca}$ & 4510 & 4499 & 2 & 1.05 \\
\hline
\end{tabular}

${ }^{\mathrm{a}}$ Source: General Electric Co.

${ }^{\mathrm{b}}$ Sample thickness

Table 21. Natural rubber, stretched $310 \%$, Ref. [24].

\begin{tabular}{|c|c|c|c|c|}
\hline Isotope/Element & $\boldsymbol{v ( H z )}$ & $\boldsymbol{v}_{\mathrm{C}}(\mathbf{H z})$ & $\boldsymbol{q}$ & $\boldsymbol{S}(\boldsymbol{\mu m})$ \\
\hline \hline $\mathrm{C}$ & 1020 & 1017 & 1 & 2.02 \\
\hline${ }^{29} \mathrm{Si}$ & 1700 & 1686 & 2 & 2.01 \\
\hline $\mathrm{Na}$ & 2100 & 2125 & 2 & 2.03 \\
\hline
\end{tabular}

\subsubsection{Natural Rubber}

Natural rubber is polyisoprene, $\left[-\mathrm{CH}_{2}-\mathrm{C}\left(\mathrm{CH}_{3}\right)=\mathrm{CH}-\mathrm{CH}_{2}-\right.$ ]$_{\mathrm{N}}$; isoprene is 2-methyl-1,3-butadiene, $\mathrm{CH}_{2}=\mathrm{C}\left(\mathrm{CH}_{3}\right)$ $\mathrm{CH}=\mathrm{CH}_{2}$. Data from an early Fitzgerald spectrum of natural rubber are listed in Table 21 [24]. Natural rubber crystallizes upon stretching above $300 \%$, as determined by x-ray diffraction. In stretched natural rubber, $S$ was measured experimentally by light scattering as $2.0 \mu \mathrm{m},[25,26]$ from which a single carbon atom resonance was calculated [27] at $1040 \mathrm{~Hz}$ by Eq. (2) which compared well with an experimentally determined resonance frequency, $1020 \mathrm{~Hz},[24]$ but the entire spectrum is analyzed in the Table 21. The listed impurities
Table 22. Teflon ${ }^{\circledR}$ at $100 \mathrm{~g}$ tension. Ref. $[32,33]$.

\begin{tabular}{|c|c|c|c|c|c|}
\hline Element & $\boldsymbol{v}(\mathbf{H z})^{\mathbf{a}}$ & $\boldsymbol{v}_{\mathbf{C}}(\mathbf{H z})$ & $\boldsymbol{v}_{H}(\mathbf{H z})^{\mathbf{b}}$ & $\boldsymbol{q}$ & $\boldsymbol{S}(\boldsymbol{\mu} \mathbf{m})$ \\
\hline \hline $\mathrm{Co}, \mathrm{Ni}^{\mathrm{c}}$ & 345 & 346 & & 1 & 1.55 \\
\hline & 357 & & 355.2 & & \\
\hline $\mathrm{Mn}, \mathrm{Fe}^{\mathrm{c}}$ & 369 & 364 & 367.9 & 1 & 1.56 \\
\hline $\mathrm{Cr}$ & 390 & & 380.5 & & \\
\hline $\mathrm{V}$ & 397 & 399 & 393.3 & 1 & 1.57 \\
\hline & & & 405.9 & 1 & 1.57 \\
\hline $\mathrm{Ti}^{\mathrm{d}}$ & 421 & 425 & & 1 & 1.57 \\
\hline
\end{tabular}

${ }^{a}$ From Ref. [32].

${ }^{\mathrm{b}}$ From Ref. [33].

${ }^{c}$ Average atomic weight used.

${ }^{\mathrm{d}}$ Appears at $500 \mathrm{~g}$ tension.

are found in the latex [28-30] and/or its byproduct wastes $[28,31]$.

\subsection{Synthetic Polymers}

\subsubsection{Teflon ${ }^{\circledR}$}

Teflon ${ }^{\circledR}$ is polytetrafluoroethylene, $\left(\mathrm{C}_{2} \mathrm{~F}_{4}\right)_{\mathrm{N}}$. Tetrafluoroethylene (TFE) is produced by reacting chloroform, $\mathrm{CHCl}_{3}$, with hydrogen fluoride gas. Only a few steels can withstand HF, chief among them is Hastelloy ${ }^{\mathbb{B}}$. The "Teflon ${ }^{\mathbb{}}$ " resonances in Table $22[27,32]$ are seen to be due the metals present in Hastelloy ${ }^{\circledR}$ or similar steel. We see once again the importance of impurities and a thorough chemical analysis. Repeatedly, in this work spectra often have way more impurity resonances than resonances due to the actual material under investigation. Sometimes the main elements do not even appear in the MRD spectrum at all.

Calculated natural (elastic) frequencies by I. L. Hopkins [33] appear in column four as $\boldsymbol{v}_{H}$. He considered the entire system of the 4-inch Teflon ${ }^{\circledR}$ bar plus the two attached copper tension wires in his lengthy and very detailed calculations. He concluded as we do here that while elastic resonances may appear in the same frequency range, the existence of other (particle wave) resonances is not therefore excluded. It should be noted that his calculated frequencies are equally spaced at all tensions, and the spacing increases with increasing tension, which has never been observed for particle wave resonances and is not predicted by particle wave theory. The spacing is $12.7 \mathrm{~Hz}$ in Table 22. Also of importance is the fact that Fitzgerald's data became increasingly resolved with increasing tension, while Hopkins' calculations showed the opposite effect. Unfortunately, Hopkins' formulae did not allow calculation of higher frequency resonances due to the ever increasing accuracy requirements of certain parameters with increasing frequency. His calculations on $2 \mathrm{~S}$ Aluminum, when compared to Bodner's data at 870 psi tension yielded similar results to those for Teflon ${ }^{\circledR}$ in Table 22: some fortuitous matches to MRD experimental data (357, 369,380 , and $393 \mathrm{~Hz}$ ), one extra calculated frequency (405.9 
Table 23. Teflon ${ }^{\circledR}$. Ref. [34].

\begin{tabular}{|c|c|c|c|c|}
\hline Element & $\boldsymbol{v}(\mathbf{H z})$ & $\boldsymbol{v}_{\mathbf{C}}(\mathbf{H z})$ & $\boldsymbol{q}$ & $\boldsymbol{S}(\boldsymbol{\mu} \mathbf{m})$ \\
\hline \hline $\mathrm{Ti}, \mathrm{V}, \mathrm{Cr}, \mathrm{Mn}, \mathrm{Fe}, \mathrm{Co}, \mathrm{Ni}$ & 375 & $373^{\mathrm{a}}$ & 1 & $1.60^{\mathrm{a}}$ \\
\hline $\mathrm{F}$ & 1000 & 1020 & 1 & 1.62 \\
\hline $\mathrm{Ti}, \mathrm{V}, \mathrm{Cr}, \mathrm{Mn}, \mathrm{Fe}, \mathrm{Co}, \mathrm{Ni}$ & 1500 & $1490^{\mathrm{a}}$ & 2 & $1.60^{\mathrm{a}}$ \\
\hline $\mathrm{Cl}$ & 2200 & 2186 & 2 & 1.60 \\
\hline
\end{tabular}

${ }^{a}$ Average atomic weight of 52 used in calculation of $S$ and $v_{c}$.

Table 24. Polyethylene from Ref. [12].

\begin{tabular}{|c|c|c|c|c|}
\hline Element/Compound & $\boldsymbol{v}(\mathbf{H z})$ & $\boldsymbol{v}_{\mathbf{C}}(\mathbf{H z})$ & $\boldsymbol{q}$ & $\boldsymbol{S}(\boldsymbol{\mu} \mathbf{m})$ \\
\hline \hline $\mathrm{Ti}_{2}\left(\mathrm{C}_{2} \mathrm{H}_{4}\right)_{2} \mathrm{Cl}_{6}{ }^{\mathrm{a}}$ & 24.3 & 25.0 & 1 & 2.37 \\
\hline $\mathrm{Ti}_{\left(\mathrm{C}_{2} \mathrm{H}_{5}\right) \mathrm{Cl}_{3}}$ & 52.0 & 49.7 & 1 & 2.29 \\
\hline $\mathrm{C}_{2} \mathrm{H}_{4} \mathrm{Cl}_{2}$ & 89.0 & 92.1 & 1 & 2.38 \\
\hline $\mathrm{C}_{2} \mathrm{H}_{5} \mathrm{Cl}$ & 134 & 141 & 1 & 2.40 \\
\hline $\mathrm{Ti}$ & 194 & 190 & 1 & 2.32 \\
\hline $\mathrm{Cl}$ & 262 & 257 & 1 & 2.32 \\
\hline $\mathrm{Al}$ & 337 & 338 & 1 & 2.34 \\
\hline $\mathrm{MgEtCl}$ & 426 & 410 & 2 & 2.30 \\
\hline
\end{tabular}

${ }^{\text {a }}$ Structurally, $\mathrm{Cl}_{3} \mathrm{Ti}\left(\mathrm{CH}_{2} \mathrm{CH}_{2} \mathrm{CH}_{2} \mathrm{CH}_{2}\right) \mathrm{TiCl}_{3}$

$\mathrm{Hz}$ ), and some MRD experimental frequencies unaccounted for by elastic theory $(345,397$, and $421 \mathrm{~Hz})$. In contrast, the Fitzgerald Theory predicts all experimental frequencies except the elastic frequencies calculated by Hopkins (357 and $380 \mathrm{~Hz}$ ).

Additional Teflon ${ }^{\circledR}$ data of Fitzgerald [34], Table 23, showed resonances at higher frequencies due to fluorine and chlorine. The latter comes from chloroform in the synthesis of TFE. In this case the individual metal resonances were not resolved as in Table $\mathbf{2 2}$ but were contained in broad peaks.

\subsubsection{Polyethylene}

Polyethylene data of Bodner [12] is presented in Table 24. Ethylene is polymerized via the Ziegler-Natta Process, which employs a complex catalyst of $\mathrm{Al}\left(\mathrm{C}_{2} \mathrm{H}_{5}\right)_{3}, \mathrm{TiCl}_{3}$ and $\mathrm{TiCl}_{4}$ on a $\mathrm{MgCl}_{2}$ support. The impurities in Table 24 are all from the catalyst system. The titanium moieties are the result of premature chain terminations. We see here the occurrence of large molecular "particles". There is no reason why entrained molecules and molecular ions cannot enter the fieldfree region to become particle waves, so long as the energy required to do so is less than any bond energy in the molecule. Fitzgerald found only a single resonance at $2750 \mathrm{~Hz}$ in the range of $100-4500 \mathrm{~Hz}$ [34]. Since corresponding details of such as molecular weight range and crystallinity are not known for both samples, a comparison is not possible. Fitzgerald's sample was under $1.0 \%$ compression, but Bodner's was under no load. Subsequent loading of Bodner's sample
Table 25. Poly(vinyl stearate) from Ref. [34].

\begin{tabular}{|c|c|c|c|c|}
\hline Molecule & $\boldsymbol{v}(\mathbf{H z})$ & $\boldsymbol{v}_{\mathrm{C}}(\mathbf{H z})$ & $\boldsymbol{q}$ & $\boldsymbol{S}(\boldsymbol{\mu} \mathbf{m})$ \\
\hline \hline Stearlyethyl benzoate & 120 & 120 & 1 & 0.980 \\
\hline stearic acid & 185 & 183 & 1 & 0.974 \\
\hline Stearlyethyl benzoate & 490 & 481 & 2 & 0.970 \\
\hline benzene & 650 & 666 & 1 & 0.992 \\
\hline stearic acid & 720 & 731 & 2 & 0.987 \\
\hline hydroxyethyl benzoate & 1235 & 1252 & 2 & 0.986 \\
\hline cumene hydroperoxide & 1400 & 1366 & 2 & 0.968 \\
\hline Ethyl radical & 1790 & 1789 & 1 & 0.979 \\
\hline
\end{tabular}

produced results similar to the no load case. Reasons for the different behavior of these samples remain unknown.

\subsubsection{Poly (Vinyl Stearate)}

Polymerization of vinyl stearate occurs via a free radical mechanism. Numerous free radical initiators are possible, including benzoyl hydroperoxide, benzoyl peroxide, ditertiary butyl peroxide, tertiary butyl hydroperoxide, diacetyl peroxide, diethyl peroxycarbonate, cumene hydroperoxide, and others. Add to this the possible solvents: methyl chloride, methylene chloride, chloroform, benzene, toluene, xylene, cyclohexane, n-heptane, ethyl ether, and dioxane, among others. From among the many combinations of initiator and solvent, the best fit of impurities to PVS data [34] is found in Table 25. Note the consistent occurrence of the benzoyl/benzoate molecular fragment as well as stearyl/stearic. Benzene would have been the logical solvent for either benzoyl hydroperoxide, benzoyl peroxide, or cumene hydroperoxide.

\section{PREVIOUS CRITICISMS OF THE FITZGERALD THEORY}

One of Fitzgerald's critics, R. W. Leonard, made the argument [35] that he could produce normal (elastic) resonances in the audiofrequency range with an $8 \mathrm{ft}$ lead rod, and therefore there need be no other explanation for resonances in this frequency range. He believed the Fitzgerald resonances to be artifacts of the apparatus, especially since so many occurred in the $2800-3000 \mathrm{~Hz}$ window.

Table 26 gives Leonard's data $\left(v_{o b s}\right)$ read off his semi log strip chart as reproduced in Refs. [21] and [35]. Standing waves were assigned in column 1 , and the speed of sound $\left(C_{S}\right)$ was calculated using the standing wave formulae, Eqs. (4) and (5). A consistent value of $C_{S}$ was obtained $\left(C_{S a v}=\right.$ $1041 \mathrm{~m} / \mathrm{s}$ ), compared to the value of $1190 \mathrm{~m} / \mathrm{s}$ for annealed lead from Ref. [36]. Thus it is proven that these are natural frequencies due to standing wave modes. However, the appearance of an additional resonance at $205 \mathrm{~Hz}$ is attributed to a particle wave mode $(q=1)$, with additional such modes, $q=2,3$, coincident with the fourth $(850 \mathrm{~Hz})$, and ninth 
Table 26. Lead resonances from Ref. [35].

\begin{tabular}{|c|c|c|c|c|c|c|}
\hline$\lambda^{\mathrm{a}}$ & $\begin{array}{c}v_{o b s} \\
(\mathbf{H z})\end{array}$ & $\begin{array}{c}v_{c}^{E} \\
(\mathbf{H z})^{\mathbf{b}}\end{array}$ & $\begin{array}{c}v_{c}^{P} \\
(\mathbf{H z})^{\mathrm{c}}\end{array}$ & $\begin{array}{c}C_{S} \\
(\mathbf{m} / \mathbf{s})^{d}\end{array}$ & $q$ & $S(\mu \mathrm{m})^{\mathrm{e}}$ \\
\hline & 205 & & 209 & & 1 & 1.08 \\
\hline $2 \mathrm{~L} / 1$ & 225 & 213 & & 1049 & & \\
\hline $2 \mathrm{~L} / 2$ & 430 & 427 & & 1024 & & \\
\hline $2 \mathrm{~L} / 3$ & 630 & 640 & & 1036 & & \\
\hline $2 \mathrm{~L} / 4$ & 850 & 854 & 838 & 1034 & 2 & 1.06 \\
\hline $2 \mathrm{~L} / 5$ & 1060 & 1067 & & 1040 & & \\
\hline $2 \mathrm{~L} / 6$ & 1280 & 1281 & & 1038 & & \\
\hline $2 \mathrm{~L} / 7$ & 1490 & 1494 & & 1036 & & \\
\hline $2 \mathrm{~L} / 8$ & 1700 & 1707 & & 1030 & & \\
\hline $2 \mathrm{~L} / 9$ & 1900 & 1921 & 1885 & 1024 & 3 & 1.07 \\
\hline $2 \mathrm{~L} / 10$ & 2100 & 2134 & & 1097 & & \\
\hline
\end{tabular}

${ }^{\mathrm{a}} \mathrm{L}=8 \mathrm{ft}$

${ }^{\mathrm{b}}$ Calculated from Eq.(6), $C_{S}=C_{S a v}=1041 \mathrm{~m} / \mathrm{s}$.

${ }^{c}$ Calculated from Eq.(4), $S=S_{a v}=1.07 \mu \mathrm{m}$.

Calculated from Eq.(6).

${ }^{\mathrm{e}}$ Calculated from Eq.(5).

Table 27. Summary of occurrence of elements in $2790-2990 \mathrm{~Hz}$ range.

\begin{tabular}{|c|c|}
\hline Element & Occurrences \\
\hline \hline $\mathrm{Cu}$ & 10 \\
\hline $\mathrm{Fe}$ & 3 \\
\hline $\mathrm{K}$ & 3 \\
\hline $\mathrm{Ni}$ & 2 \\
\hline $\mathrm{Ti}$ & 1 \\
\hline $\mathrm{Li}$ & 1 \\
\hline $\mathrm{O}$ & 1 \\
\hline $\mathrm{Ge}$ & 1 \\
\hline None & 18 \\
\hline TOTAL $^{\mathrm{a}}$ & $38^{\mathrm{b}}$ \\
\hline
\end{tabular}

${ }^{\text {a }}$ No resonances in the $2790-2890 \mathrm{~Hz}$ range.

${ }^{\mathrm{b}}$ In two samples of 38 there were two elements in the range.

$(1900 \mathrm{~Hz})$ standing wave modes, respectively. These three inelastic modes give a consistent value of $S(\operatorname{Sav}=1.07 \mu \mathrm{m})$ from Eq. (3). Elastic frequencies, $v_{c}^{E}(\mathrm{~Hz})$, are calculated from Eq. (4) using $C_{S a v}=1041 \mathrm{~m} / \mathrm{s}$. Particle wave frequencies, $v_{c}^{p}(\mathrm{~Hz})$, are calculated from Eq. (2) and $S_{a v}$ as in all the above Tables.

$C_{S}=v \lambda$
Table 28. Alnico magnet, Ref. [38].

\begin{tabular}{|c|c|c|c|c|}
\hline Element & $v(\mathbf{H z})$ & $\boldsymbol{v}_{\mathrm{C}}(\mathrm{Hz})$ & $\boldsymbol{q}$ & $\boldsymbol{S}(\boldsymbol{\mu m})$ \\
\hline \hline \multicolumn{5}{|c|}{1 inch steel ball } \\
\hline Co & 1829 & 1826 & 2 & 1.36 \\
\hline $\mathrm{Co}$ & 4104 & 4110 & 3 & 1.36 \\
\hline \multicolumn{5}{|c|}{0.687 inch steel ball } \\
\hline $\mathrm{Ti}$ & 2059 & 2043 & 2 & 1.42 \\
\hline $\mathrm{Co}$ & 6585 & 6637 & 4 & 1.43 \\
\hline
\end{tabular}

$\lambda=2 L / N N=1,2, \ldots$

Thus it is seen that a large enough object ( $8 \mathrm{ft} !)$ can give elastic mode resonances in the audiofrequency range, but that, in spite of some overlaps, these are distinct from the inelastic particle wave resonances in the same range and in no way disprove the existence of the latter.

In arguing that the Fitzgerald resonances were instrumental artifacts, Leonard stated [35] that "The results on all hard crystalline materials yield a resonance at a frequency very near $2890 \mathrm{cps}$... ." (emphasis ours) We summarize our results by element for the range $2790-2990 \mathrm{~Hz}(2890 \pm 3.5 \%)$ in Table 27. In only 20 (not "all") of 38 samples analyzed here were resonances found in that range. Copper was responsible in 10 cases, but in all of these copper was a major constituent or known impurity in the sample. Iron was the next most commonly observed element (three samples). Since copper and iron are components of the apparatus, the suspicion of Leonard might be warranted. However, in 18 of 38 samples no resonance was found in the target range. Due to the variety of elements in Table 27, many of which are not found in the seven different apparati used, and the 18 occurrences of no resonances at all in the target range, it seems unlikely that an apparatus constant (causing resonance at near $2890 \mathrm{~Hz}$ ) is involved.

Leonard also noted that fused quartz is amorphous, while the Fitzgerald Theory predicts resonances for crystalline solids with distinct rows of equally spaced atoms. More recent research [37] shows that fused quartz has medium range order, $20 \AA$, which might be preferentially aligned to allow for much longer linear ordering. Obviously, a complete study of all forms of $\mathrm{SiO}_{2}$, including glasses, with measured impurities would be revealing. It is proposed here that crystallinity is not required for these resonances, only a field free region which extends along a line without interruption of fixed particles. As noted below, more research in amorphous and partially ordered materials is recommended.

Another of Fitzgerald's critics, I. L. Hopkins, measured resonances of steel balls of various diameters in the audiofrequency range, and like Leonard he concluded that quantum mechanics was not necessary to explain elastic phenomena in the audiofrequency range [38, 39]. However, calculations in Table 28 indicate that the alnico magnet driver in his apparatus may be the source of inelastic resonances of the Fitzgerald type at these frequencies. 


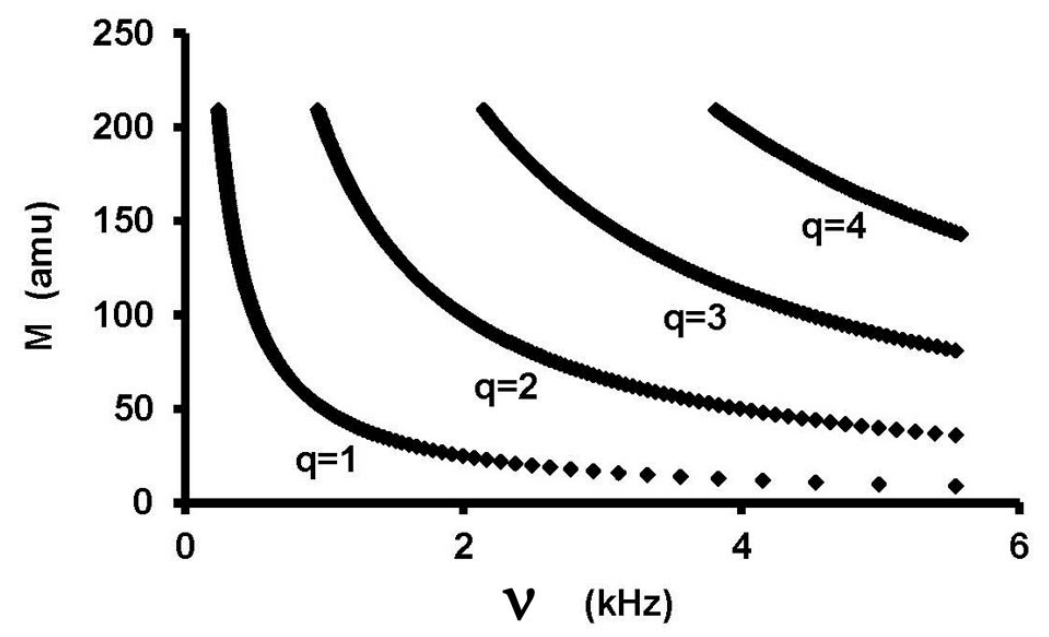

Fig. (1). M vs. $v$, Eq. (6).

The composition of alnico (Al-Ni-Co) alloys is typically $8-12 \% \mathrm{Al}, 15-26 \% \mathrm{Ni}, 5-24 \% \mathrm{Co}$, up to $6 \% \mathrm{Cu}$, up to $1 \%$ $\mathrm{Ti}$, and the balance $\mathrm{Fe}(31-72 \%)$. Precipitation hardening in the production process can cause residual stress in the host lattice, either tensile or compressive. Either could affect the value of $S$. Resonances could be due to either host or precipitate phase, which might have different mosaic sizes and hence different values of $S$. In view of this, the assignments in Table 28 must be considered tentative. The case of the 0.687 inch ball is a bit unusual in that lower acoustic modes $(q=2,3)$ for Co were apparently not observed, although Hopkins admits that the alnico magnet is part of the same resonant system as the steel ball, the base plate, the solenoid, and the rest of his apparatus, all of which contribute resonances in the same range, and that filtering out the relevant ones is not straight forward. We do not know which resonances he may have discarded and not reported. In any case, Fitzgerald never measured resonances in steel balls, so these experiments are hardly relevant to the particle wave theory. And, except for the Federal Communications Commission, nobody owns the audiofrequency band.

\section{SUMMARY OF RESULTS}

(1) Interpreting MRD spectra of 38 widely different samples - single crystal, polycrystalline - metals, salts, natural and synthetic polymers - according to the particle wave theory of E. R. Fitzgerald has shown excellent agreement between measured and calculated resonance frequencies by Eq. (2).

(2) Standard deviations from the average value of crystal mosaic size calculated for each frequency within a sample phase by Eq. (3) varied from $0.08 \% S_{a v}$ to $1.78 \% S_{a v}$, with a mean of $0.77 \% \mathrm{~S}_{\mathrm{av}}$ for 41 sample phases. By comparison, for $\mathrm{Ca}$ and $\mathrm{P}$ in human cancellous bone, the corresponding value was $2.27 \% S_{a v}$ for ${ }^{40} \mathrm{Ca}$ and $\mathrm{P}[6]$ and $0.64 \% \mathrm{~S}_{\mathrm{av}}$ for ${ }^{44} \mathrm{Ca}$ and $\mathrm{P}$ (alternate interpretation) [8].
(3) The average \% difference, $100\left|v-v_{c}\right| / v_{c}$, for 238 resonance frequencies in 41 sample phases was $1.3 \%$. Of these, $189(79 \%)$ were less than $2 \%$.

(4) Overall, the majority of resonances were the result of impurities in the lattice. The great majority of these was either documented in the original sample analyses or was highly likely due to chemical similarities, and/or sample origin, and/or sample processing.

(5) Fig. (1) shows mass numbers $M$ as a function of frequency over the range of this study $(0-5600 \mathrm{~Hz})$ for $S=1$ $\mu \mathrm{m}$, calculated from Eq. (6). $N_{0}$ is Avogadro's number. Each curve represents a different $q$-value, 1 to 4 . Frequency was chosen as the independent variable here to parallel the interpretation process. Physical reality is the opposite: mass determines frequency.

$M=\sum_{q=1}^{4} q^{2} h N_{0} /\left(8 v S^{2}\right)$

Fig. (2) shows the number of mass numbers within a \pm $2 \%$ frequency window as a function of frequency according to Eq. (7) [obtained by differentiating Eq. (6)], with $|\Delta v| / v=0.02$.

$|\Delta M|=\sum_{q=1}^{4} \frac{q^{2} h N_{0}}{8 v S^{2}}\left(\frac{|\Delta v|}{v}\right)$

Again, $S=1 \mu \mathrm{m}$. The discontinuities are the result of sequential addition of $q$-values from 1 to $(1$ and 2$)$ to ( 1 and 2 and 3 ) to (1 and 2 and 3 and 4) with increasing frequency.

From Figs. (1 and 2) it is apparent that there are multiple choices of isotopic masses to satisfy Eq. (2) within a $\pm 2 \%$ frequency window and that the number of choices increases with increasing frequency. This demonstrates the need for 


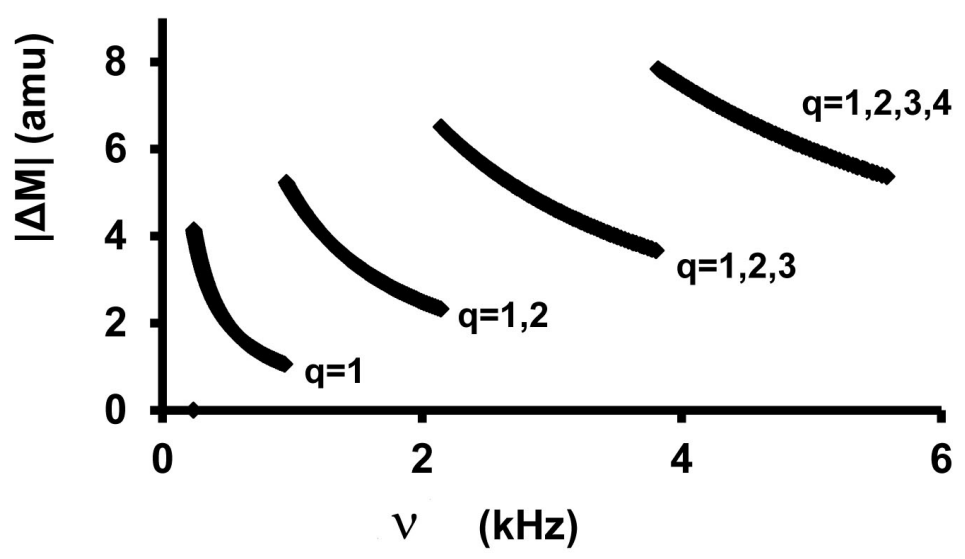

Fig. (2). $|\Delta \mathrm{M}|$ in a $\pm 2 \%$ frequency window $v s . v$, Eq. (7).

very complete and highly sensitive chemical analyses to guide interpretation. In this work, the (arbitrary) $\pm 2 \%$ frequency window was met for 195 of 238 experimental frequencies. Better fits were possible in some cases, but only by introducing unlikely elements as impurities. Only about a fourth of all elements are "common," which constrains realistic assignments.

(6) Previous elastic mode interpretations of some of the audiofrequency resonances in lead, $2 \mathrm{~S}$ Aluminum and in Teflon $^{\circledR}$ failed to predict all the frequencies and no more. In these cases the particle wave theory predicted all the nonelastic frequencies (including overlaps with elastic frequencies) and no more. Only in the alnico samples did elastic theory predict all the frequencies (but only 2 in each of 2 samples) and no more in the frequency range of the experiment, but even here some frequencies were apparently discarded.

\section{CONCLUSION}

The following conclusions are drawn from the present work and careful reading of previously reported work:

(1) The Particle Wave Theory of plastic deformation in crystalline solids has been upheld by detailed quantitative interpretations of past MRD spectra of 38 samples obtained by six different research individuals or groups operating independently with seven different apparati.

(2) In larger samples, elastic normal modes are found in the audiofrequency range, where they occasionally overlap particle wave frequencies. Alternate interpretations according to various elastic theory expressions are either partially coincidental and inadequate as shown here or flawed as suggested by Fitzgerald [21].

(3) In addition to atoms and monatomic ions, interpretation of some spectra requires that molecules or molecular ions be proposed, especially for very low frequency resonances, necessitating a high mass particle (Eq. 2).

(4) In complex, multiphase systems such as some alloys, multiple crystal mosaic sizes, $S$, corresponding to different phases, are necessary to interpret the spectra. This applies in particular to precipitation hardened alloys.

(5) Fitzgerald's requirement that for particles to emerge in the field-free region they must be aligned in a row of evenly spaced like isotopes [8], seems unnecessary. The large number of isotopes and impurities in some materials and the imposition of maximum entropy (which favors mixing over segregation) make this segregation improbable or impossible. In fact, a simple particle-in-a-box model, without reference to a lattice, would appear to suffice for the interpretation of MRD spectra. This would also allow for a particle-wave interpretation of spectra of some amorphous or only slightly ordered structures with lengthy, open, field-free regions.

(6) The overall simplification of spectra with time, with increased static stress, and with annealing suggests that, under the influence of periodic shear stress, impurities move out of the primary phase into precipitates, dislocations, or grain boundaries. In this view, the odd substituent or interstitial atom would be more likely to enter the field free region because it would be out of phase (and hence in a higher energy state) with lattice vibrations, due to its different mass.

\section{RECOMMENDATIONS FOR FUTURE WORK}

Recommendations are made for future experiments in MRD:

(1) Repeat many of the original experiments on the same materials, this time analyzed for impurities by the current best techniques, including electron microprobe analysis (also see (3)). Keep sample size small enough to prevent interference from natural (elastic) frequencies. Measure higher frequencies (to $10 \mathrm{kHz}$ or more) [40] to confirm assignments (same element, higher $q$-value). Always measure a no load or minimum load spectrum for comparison with other results and to complete the analysis in (5) below.

(2) Measure the resonance spectra of some materials with exchangeable cations, such as clays, zeolites, and ion exchange resins. Replace the ions and redetermine the spectra 
to see if frequencies shift predictably, i.e., $v_{2} / v_{1}=m_{1} / m_{2}$ for masses $m_{1}$ of original ion and $m_{2}$ of replacement ion. We will then know for certain the origin of both frequencies, $v_{1}, v_{2}$.

(3) Integrate modern microscopy techniques on the same samples to determine distribution of impurities, their composition, and crystal mosaic size, before and after prolonged periodic stressing.

(4) Measure complete spectra in far less time than was required in the past (minutes instead of days or weeks) employing, for example, a more recent apparatus such as described in [40]. If equilibration is slow, this may not be possible, but it would open the possibility of studying the dynamics of equilibration.

(5) With (1) - (4) in hand, do more detailed time studies on changing samples to determine the fate of impurity particles under periodic stress (e.g., do they migrate to dislocations or grain boundaries, and do they form separate phases?). The application of periodic audiofrequency stress is a potential means of strengthening materials and otherwise affecting their properties, because it affects the distribution of impurities.

(6) Investigate amorphous and partially ordered materials, including quasi-crystals, to determine whether crystallinity is an absolute requirement for particle wave resonances.

(7) Confirm and explain the release of $\mathrm{P}$ atoms in bone apatite and $\mathrm{Si}$ and other atoms in quartz where they are tightly bonded to four oxygen atoms.

(8) Investigate the interaction of electromagnetic induction and radiation with solids exhibiting mechanical resonance dispersion. Potentially this could lead to detection and identification of materials at great distances, up to km. Further, because of its long wavelength, radiation in this audiofrequency region penetrates all common materials except metals, yielding opportunities for detection of hidden objects.

\section{CONFLICT OF INTEREST}

The author confirms that this article content has no conflict of interest.

\section{ACKNOWLEDGEMENTS}

Declared none.

\section{REFERENCES}

[1] Fitzgerald JW, Fitzgerald ER. Measurements of the complex dynamic shear compliance and loss tangent of pilot whale blubber and the role of blubber in flow-noise reduction. J Acous Soc Am 1990; 87(S1): S147.

[2] Hutchins CM, Hutchins MA, Fitzgerald ER. Dynamic mechanical properties of violin wood and the tone qualities of violins. I: Audiofrequency complex shear compliance, modulus, and shear sound velocity and attenuation measurements. J Acous Soc Am 1993; 93(4): 2381.

[3] Fitzgerald ER. Mechanical resonance dispersion in metals at audiofrequencies. Phys Rev 1957; 108(3): 690-706.
[4] Fitzgerald ER. Momentum transfer in crystal lattices with vibrating atoms. Int J Theory Phys 1969; 2(1): 41-58.

[5] Fitzgerald ER, Tsai J. The Schroedinger Equation and momentum transfer in crystals. Int J Sol Struct 1967; 3: 927-33.

[6] Pugh W, Rose RM, Paul IL, Radin EL. Mechanical resonance spectra in human cancellous bone. Science 1973; 181: 271-2.

[7] Black J. Comment on "Mechanical resonance spectra in human cancellous bone." Science 1973; 181: 273.

[8] Fitzgerald ER. Calcium-isotope effects in mechanical spectra of cancellous bone. Med Biol Eng 1975; 13(5): 717-9.

[9] Fitzgerald ER. Audio-Frequency Mechanical Spectra of a Butadiene Terpolymer. J Appl Polym Sci 1975; 19: 2015-31.

[10] Lide DR, Ed. CRC Handbook of Chemistry and Physics. $88^{\text {th }}$ ed. Boca Raton: CRC Press, 2008; pp. 1-7 - 1-8.

[11] Lide DR, Ed. CRC Handbook of Chemistry and Physics. $88^{\text {th }}$ ed. Boca Raton: CRC Press, 2008; pp. 1-9 - 1-12.

[12] Bodner SR. On anomalies in the measurement of the complex modulus. Trans Soc Rheol 1960; 4: 141-58.

[13] Gingerich KA, Cocke DL, Miller F. Thermodynamic investigation of the lead molecules $\mathrm{Pb}_{2}, \mathrm{~Pb}_{3}$, and $\mathrm{Pb}_{4}$ by mass spectrometry. $\mathrm{J}$ Chem Phys 1976; 64(10): 4027-33.

[14] Bodner SR. A Theory for the occurrence of intrinsic resonances in stressed solid materials. Trans Soc Rheol 1961; 5(1): 297-316.

[15] Hilpert K, Gingerich KA. Atomization enthalpies of the molecules $\mathrm{Cu}_{3}, \mathrm{Ag}_{3}$, and $\mathrm{Au}_{3}$. Ber Bunsenges. Phys Chem 1980; 8(8): 739-45.

[16] Wang C-F, Peng S-M, Chan C-K, Che C-M. Luminescent silver(I) cluster. Synthesis, spectroscopic properties and X-ray crystal structure of $\left[\mathrm{Ag}_{3}(\mathrm{C} \equiv \mathrm{CPh})_{2}(\mathrm{dppm})_{3}\right] \mathrm{Cl} \cdot 4 \mathrm{Et}_{2} \mathrm{O} \cdot \mathrm{H}_{2} \mathrm{O}\{\mathrm{dppm}=$ bis (diphenylphosphino)methane \}. Polyhedron 1996; 15(11): 1853-8.

[17] Fitzgerald ER. Simple method for observing audiofrequency resonances and sound beams in crystals. J Acous Soc Am 1964; 36(11): 2086-9.

[18] Fitzgerald ER. Mechanical resonance dispersion in single crystals. Phys Rev 1958; 112(4): 1063-75.

[19] Molinari E. Treatise on General and Industrial Organic Chemistry. $2^{\text {nd }}$ ed. Pope TH, translator. Philadelphia: Forgotten Books 1913; $336 \mathrm{ff}$.

[20] Gotsky ER, Stearns CA. Mechanical resonance dispersion and stress-strain behavior of several ionic crystals, NASA Technical Note D-2566. Washington: National Aeronautics and Space Administration: 1965 .

[21] Fitzgerald ER. Observations of nonelastic audiofrequency resonances. J Acous Soc Am 1966; 39(5)(1): 870-7.

[22] Goldberg ED. The composition of sea-water. In: Hill MN, Ed Comparative and descriptive oceanography. Cambridge, Mass: Harvard University Press 2005; p. 4.

[23] Fitzgerald ER. Mechanical resonance dispersion in quartz at audiofrequencies. Phys Rev 1958; 112(3): 765-84.

[24] Fitzgerald ER. Dynamic mechanical properties of stretched natural rubber. J Acous Soc Am 1961; 33: 1305-14.

[25] Yau W, Stein RS. Light scattering from crystalline superstructure in stretched natural rubber. Polym Lett 1964; 2(2): 231-6.

[26] Yau W, Stein RS. Proceedings of the American Physical Society. Annual Meeting 1963. Bull Am Phys Soc 1963; 8: 241.

[27] Fitzgerald ER. Particle waves and audiofrequency modes in crystals. J Acous Soc Am 1966; 39(5)(1): 856-69.

[28] John CK. Wastes originating from agriculture and forestry. In: Lectures Presented at the Fifth SAO/SIDA Workshop. Sweden: Swedish Funds Trust TF-RAS 34 (SWE), 1977; (Suppl 1): pp. 113115.

[29] Archer BL, Barnard D, Cockbain EG, Dickenson PB, McMullen AI. Structure, composition, and biochemistry of Hevea latex. In: Bateman L, Ed. The chemistry and physics of rubber-like substances. London: McLaren and Sons Ltd 1963; pp. 41-61.

[30] Ng SK, Lai PT. Simultaneous determination of copper, manganese, iron, and calcium in natural rubber by emission spectrography. Appl Spectrosc 1972; 26(3): 369-72.

[31] John CK. Non-rubber constituents in Hevea latex and their possible utilization. In: Stanton WR, Ed. Waste recovery by microorganisms. Kuala Lumpur: The Ministry of Education of Malaysia 1972; pp. 110-7.

[32] Fitzgerald ER, Woodward AE. Mechanical resonance dispersion in polytetrafluorethylene rods in transverse vibration. Kolloid Z 1960; 172(2): 177-81. 
[33] Hopkins IL. Comments on the experiments of Bodner and of Fitzgerald and Woodward on laterally vibrating beams under axial tension. Trans Soc Rheol 1966; 10(1): 51-72.

[34] Fitzgerald ER. Mechanical resonance dispersion in crystalline polymers at audio-frequencies. J Chem Phys 1957; 27(5): 1180-93.

[35] Leonard RW. Comment on the existence of the Fitzgerald effect. J Acous Soc Am 1965; 38: 673

[36] Lide DR, Ed. CRC Handbook of Chemistry and Physics. $88^{\text {th }}$ ed. Boca Raton: CRC Press 2008; 14-39.
[37]

Received: June 02, 2014

(c) Haskell V. Hart; Licensee Bentham Open.

This is an open access article licensed under the terms of the Creative Commons Attribution Non-Commercial License (http://creativecommons.org/licenses/by-nc/3.0/) which permits unrestricted, non-commercial use, distribution and reproduction in any medium, provided the work is properly cited. 